atomic structure, emphasizing the importance of recent work on positronium; and F. G. Brickwedde explains the curious physical and statistical properties of the quantum liquid, helium II. V. F. Weiskopf analyses the complicated pattern of the elementary particles, and forecasts more to come as cosmotron work in the ultra-high energy range develops. 'There are three contributions of a more general typeRichard Courant on the many applications of numerical methods, with the strong-focusing synchrotron, weather forecasting, and flood control as typical examples; P. M. Morse on the adaptation of operational research to linear programming in business, and the uses of the Monte Carlo method; and E. U. Condon, on physics and the engineer, directing attention to the shortage of high-school science teachers. This last appears to be a feature of the times, and not restricted to any one country; one wonders if it is really the only problem that is quite unapproachable by the methods of operational research, for there must be some way of averting the impending academic dust-bowl.

Each article starts with a brief elementary intro. duction, and the general reader might gain from the book a good idea of what physicists have been doing recently, more particularly in the United States. But it is primarily intended for the scientific reader who wishes for a broad and fairly detailed picture of current progress. He will find it outstandingly useful.

G. R. Noakes

\section{THERMODYNAMICS OF BIOLOGICAL SYNTHESIS}

\author{
Some Principles of Energetics in Biochemical \\ Reactions
}

By Prof. Irving M. Klotz. Pp. vii +64. (New York : Academic Press, Inc.; London: Academic Books, Ltd., 1957.) 3 dollars.

CEVERAL selected topics of thermodynamics are $S$ of importance for the interpretation of the results of biochemical experiments. A closer understanding of their principles is helpful to biochemists for such practical purposes as well as for a strategic view of problems of the maintenance and development of biological systems. Only 20-25 years ago there were still lively disputes among eminent scientists as to whether the laws of thermodynamics are strictly applicable to biological systems. Some suggested that evolution or man's intellect had special 'Maxwell demons' to enable them to contravene the second law of thermodynamics. Good expositions of the thermodynamies of open systems and of the relations between information (intellect) and entropy have converted most people to the only acceptable contention that the laws of thermodynamics are applicable to biological phenomena.

Classical chemical thermodynamics was developed in a form most suitable for the interpretation of the results of experiments of isolated chemical systems at equilibrium and under standard conditions. Biochemical reactions in a cell, or in one of the many multi-enzyme systems used for the study of synthetic processes, are not in equilibrium but in a steady state. The composition of the solution must be suitable for the reaction, and cannot be varied to suit the convenience of physical interpretation. Thermodynamic properties of substances of biochemical importance can, of course, be determined under standard equilibrium conditions, but a study of the special aspects of the energetics of biochemical reactions requires the consideration of special conditions.

In Dr. Klotz's little book none of these problems of importance to biochemists is considered. $\mathrm{He}$ presents the most elementary exposition of thermodynamies I have ever seen, and illustrates it with examples from reactions of protein molecules and of biochemically important esters. As such this book should be very useful for students taking a first-year course in a biological subject; no previous knowledge of physics or physical chemistry is required, but the relevance of phosphate transfer should be understood. Even for such a junior reader the first few pages are somewhat unsophisticated, and it is my experience that somewhat greater rigour in the derivation of thermodynamic functions makes it easier for students to understand and remember them.

Apart from the subject-matter mentioned above, Dr. Klotz gives a good elementary account of the thermodynamic principles of electrode processes, osmotic pressure, sedimentation equilibria and of the molecular-statistical basis of thermodynamies. One must not look to this book for thermodynamic data for the reactions discussed ; those given are not carefully chosen nor are adequate references to the literature given. Another very serious omission is that nowhere in the book can one find a discussion of the effect of $p H$ on the reactions of charged com. pounds. Ionizable groups are involved either directly or indirectly in most biochemical reactions and consequently a brief treatment of the relation between dissociation constants, hydrogen ion concentration and chemical equilibria does seem essential. One must point out that the so-called standard free energies quoted for various hydrolysis reactions are only valid at definite $p H$ values, which are not quoted in the book.

All these remarks should not be taken as a severe criticism of the book; different readers would expect different topics discussed in sixty-four pages under this title. Unfortunately, most people in Britain will find the price prohibitive at about $4 d$. a small page ; surely this is just the sort of book which should be available cheaply between paper covers.

Herbert Gutfreund

\section{DAIRY PLANT CONTROL}

\section{Laboratory Control of Dairy Plant}

By Dr. J. G. Davis. Pp. xii + 395. (London: Dairy Industries, Ltd., 1956.) $30 s$.

T ABORATORY control of dairy plant is becoming 1 more essential nowadays with the ever-increasing tendency for larger 'through-puts' of plant. Mistakes which with small plant were unfortunate can now mean a serious economic loss with large plant. Hence strict laboratory control has now become a necessity, and, in consequence, so has a book on the subject.

The author first deals with the purpose of plant control, and underlines the fact that testing must be economic. The principles of cleaning and sterilizing plant are given a chapter as complementary information to the succeeding chapter on methods of assessing the cleanliness and sterility of dairy plant. On p. 19 the author recommends the inclusion of sodium sulphite in a detergent to minimize attack on tin 